УДК 616.12-073.97-71

\title{
Hardware-Program Complex on the Basis of Recorder MCM-11
}

\author{
Alexander V. Soldatov*a, \\ Vasily V. Cherepanov ${ }^{b}$ and Gennady M. Aldonin ${ }^{a}$ \\ ${ }^{a}$ Siberian Federal University \\ 79 Svobodny, Krasnoyarsk, 660041, Russia \\ ${ }^{b}$ Krasnoyarsk State Pedagogical University \\ named after V.P. Astafeva \\ 89 Ada Lebedeva Str., Krasnoyarsk, 660049, Russia
}

Received 29.05.2018, received in revised form 27.06.2018, accepted 13.07.2018

The hardware-software complex based on the Holter type recorder MCM-11 with the possibility of "on line" continuous remote monitoring of an electrocardiogram (ECG) and pulse wave on the tablet, smartphone, PC or laptop via Bluetooth interface is described. The remote monitoring software "ECG Manager" on the basis of "Windows" and "Android" operating systems (OS) based on the processes accumulated in the MKM-11 digital data and allows to detect latent electro-cardio signal structure using wavelet transform, which reflects phase-spatial passage of the excitation on cardiac conduction network segments.

Keywords: electrocardiogram, photoplethysmograph, monitoring, hardware-software complex, conduction of the nervous network of the heart, solitons, fractals, self-similarity, structural wavelet analysis.

Citation: Soldatov A.V., Cherepanov V.V., Aldonin G.M. Hardware-program complex on the basis of recorder MCM-11, J. Sib. Fed. Univ. Eng. technol., 2018, 11(6), 671-678. DOI: 10.17516/1999-494X-0084.

(c) Siberian Federal University. All rights reserved

This work is licensed under a Creative Commons Attribution-NonCommercial 4.0 International License (CC BY-NC 4.0).

* Corresponding author E-mail address: anvin@sibnet.ru 


\title{
Аппаратно-программный комплекс \\ на базе рекордера МКМ-11
}

\author{
А.В. Солдатов ${ }^{\mathrm{a}}$, \\ В.В. Черепанов ${ }^{\sigma}$, Г.М. Алдонин ${ }^{\mathrm{a}}$ \\ ${ }^{a}$ Сибирский федеральный университет \\ Россия, 660041, Красноярск, пр. Свободный, 79 \\ ${ }^{6}$ Красноярский государственный педагогический \\ университет им. В.П. Астафьева \\ Россия, 660049, Красноярск, ул. Ады Лебедевой, 89
}

В работе описан аппаратно-программный комплекс дистанционного мониторинга (АПК ДМ), разработанный на основе рекордера холтеровского типа МКМ-11, предназначенный для индивидуального мониторинга функиионального состояния организма (ФСО) в клинической практике, амбулаторных, бытовых и производственных условиях и в спортивной медицине. Особым качеством АПК ДМ на базе МКМ-11 является то, что он обеспечивает структурный анализ биопрочессов и биосигналов на основе положений теории самоорганизаџии методами нелинейных динамических моделей.

Ключевые слова: электрокардиограмма, фотоплетизмограмма, мониторинг, аппаратнопрограммный комплекс, проводящая нервная сеть сердия, солитоны, фракталы, самоподобие, структурный вейвлет-анализ.

\section{Введение}

Сердечно-сосудистые заболевания (СС3) имеют длительный инкубационный период и проявляются только через несколько лет после воздействия факторов риска, связанных с неблагоприятной окружающей средой или образом жизни. Необходимо совершенствование технических средств индивидуального мониторинга функционального состояния организма (ФСО) для своевременного выявления с помощью компьютерных технологий латентных форм ишемических болезней сердца (ИБС).

Постановка проблемы. Актуальной задачей мониторинга состояния сердечнососудистой системы (ССС) является создание эффективных алгоритмов компьютерных технологий обработки электрокардиосигнала (ЭКС). Для исследования ССЗ в лаборатории медицинского приборостроения Института инженерной физики и радиоэлектроники (ИИФиРЭ) Сибирского федерального университета (СФУ) разработан аппаратно-программный комплекс дистанционного мониторинга (АПК ДМ). АПК ДМ, разработанный на основе рекордера холтеровского типа МКМ-11 (рис. 1), предназначен для мониторинга ФСО в клинической практике, амбулаторных, бытовых и производственных условиях и в спортивной медицине [1,2].

Материалы и методы. Особенностью кардиомонитора МКМ-11 является наличие канала электрокардиосигнала (ЭКС) и двух каналов пульсовой волны (ПВ), для получения фотоплетизмограммы (ФПГ) с различных точек (мочка уха, фаланги пальцев рук и ног). Также предусмотрена возможность непрерывного дистанционного контроля электрокардиограммы (ЭКГ) и ПВ «online» на планшете, смартфоне, компьютере или ноутбуке через 

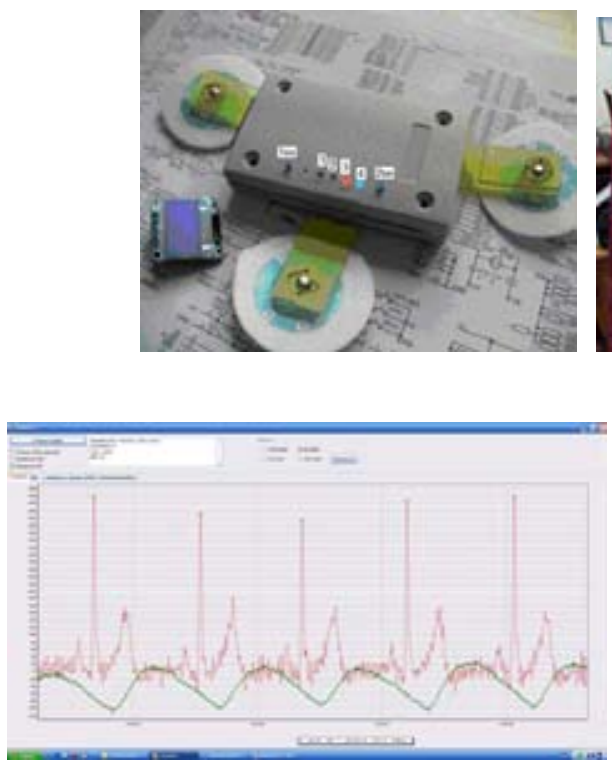

$\sigma$

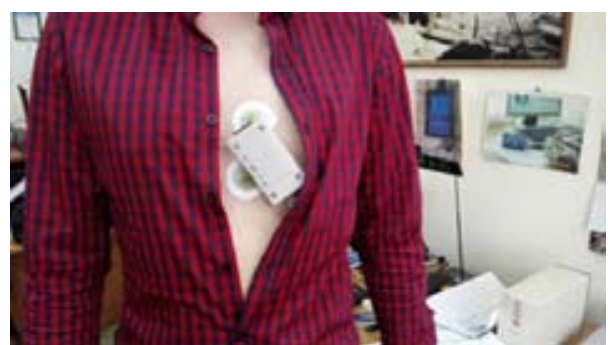

$a$

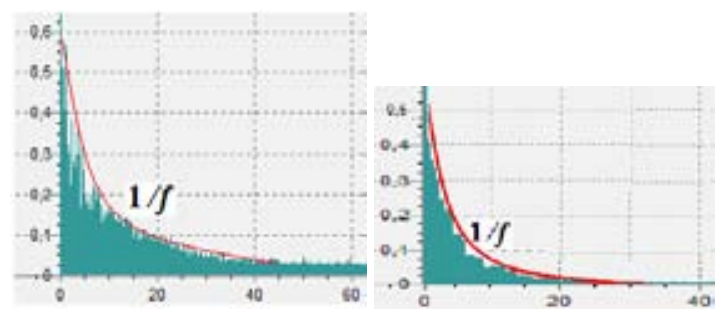

Рис. 1. Внешний вид кардиомонитора МКМ-11 (a) и записи ЭКС и ПВ (б), Фурье-спектр ЭКС и ПВ сигналов (в)

Fig. 1. Appearance of the MKM-11 cardiomonitor (a), and recording of ECS and PW (б), Fourier spectrum of ECS and PW signals ( 8 )

Bluetooth интерфейс. Также прибор позволяет записывать данные на карту памяти $(\mu \mathrm{SD})$ для последующей передачи и обработки на персональном компьютере. Благодаря своим небольшим габаритам и малому весу (не более 100 г) прибор не вызывает дискомфорта и прост в управлении.

Программное обеспечение (далее ПО) «ECGManager» для АПК ДМ на базе операционной системы (OC) «Windows» производит обработку накопленных в MКМ-11 цифровых отсчетов биосигналов, производит различные методы фильтрации сигналов, определяет их амплитуду, временные характеристики и Фурье-спектры, а также такие параметры сердечно-сосудистой деятельности, как скаттерограмма ЭКГ, время распространения пульсовой волны (ВРПВ), кардиоинтервалограмма, количество экстрасистол. ПО также обеспечивает дистанционный мониторинг ФСО в режиме «online» с МКМ-11 через Bluetooth на смартфон, планшет, ноутбук или персональный компьютер (ПК). Интерфейс программы представлен на рис. 2. ПО дает возможность инвертировать входной сигнал, использовать фильтры для подавления шумов и изменять амплитуду сигнала, меняя коэффициенты усиления.

ПО «ECGManager» на базе OC «Android» обеспечивает возможность просмотра ЭКГ, ФПГ и РПГ кровотока в режиме «online» с МКМ-11 посредством Bluetooth-соединения на любых устройствах, поддерживающих ОC «Android». Интерфейс программы представлен на рис. 3.

Данное ПО также дает возможность выводить на дисплей несколько каналов и изменять амплитуду сигнала, меняя коэффициенты усиления (рис. 4).

$$
-673-
$$




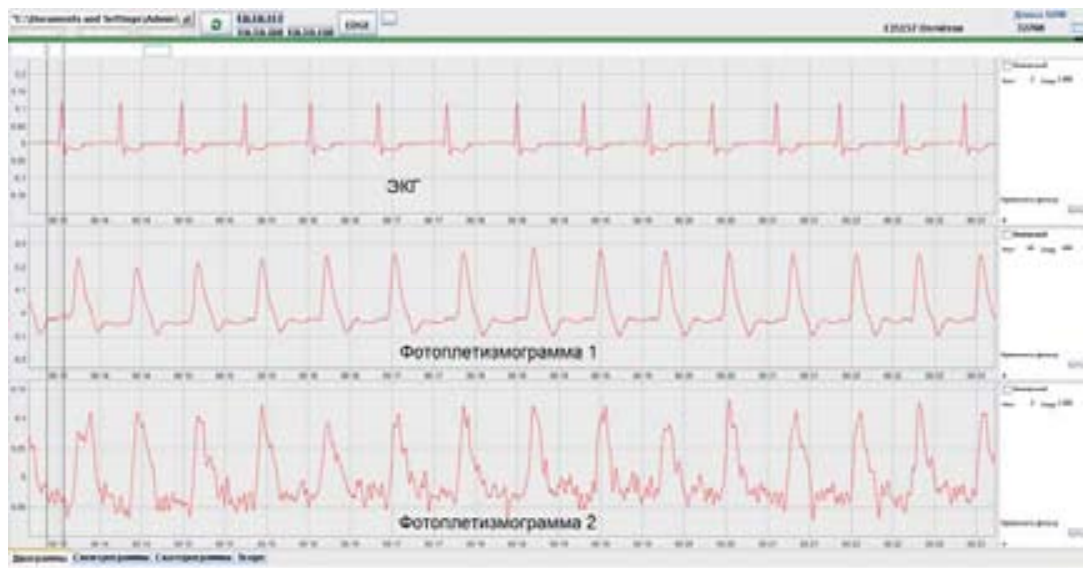

Рис. 2. Окно программы «ECGManager» для OC Windows

Fig. 2 Window of the program "ECGManager" for Windows OS

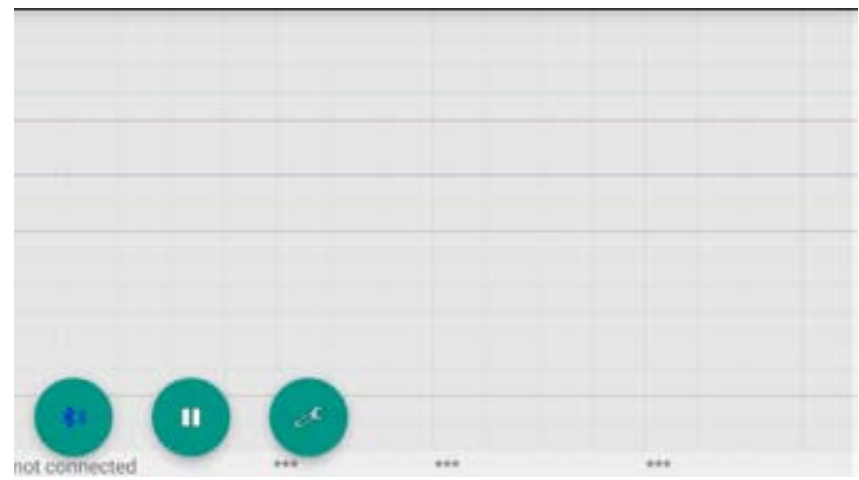

Рис. 3. Окно программы «ECGManager» на базе OC "Android"

Fig. 3 Window of the program "ECGManager" for Android OS

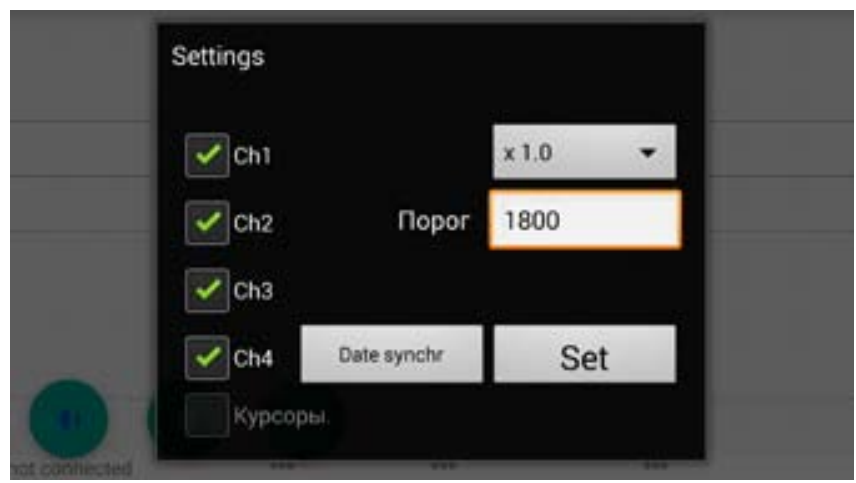

Рис. 4. Изменение амплитуды сигнала

Fig. 4. Setting range of signal amplitude 


\section{Результаты и обсуждение}

Получаемая с помощью АПК ДМ информация обеспечивает объективную оценку ФСО в процессе исследования. С помощью ПО «ЕCGManager» для рекордера МКМ-11 проводится предварительный анализ основных параметров гемодинамики ЭКГ и ПВ.

Экспериментальные исследования ССД в кардиологии и в спортивной медицине представлены на рис. 5-8.

Особым качеством АПК ДМ на базе МКМ-11 является то, что он обеспечивает структурный анализ биопроцессов и биосигналов на основе положений теории самоорганизации методами нелинейных динамических моделей [3].

Структурная организация биопроцессов и биосигналов биосистем выявляется при их Фурье- и вейвлет-преобразованиях. На рис. 2, в представлены Фурье-спектры исследуемых ЭКС и ПВ гармонического Фурье-спектра ЭКС вида $1 / f^{\beta}$, что отражает самоподобную фрактальность флуктуаций, определяемых топологией ПНСС - фрактальную структуру, ветвящуюся с самоподобием по закону Фибоначчи (рис. 9), генерирующую флуктуации в узлах ветвления ПНСС [4].

На рис. 9 представлено вейвлет-преобразование снимаемого сигнала. АПК ДМ на базе МКМ-11 обеспечивает более точное отражение процессов в ПНСС, позволяет выявлять латент-

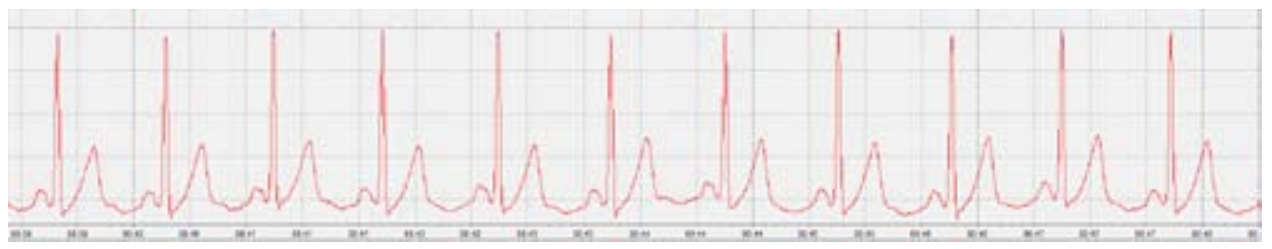

$a$

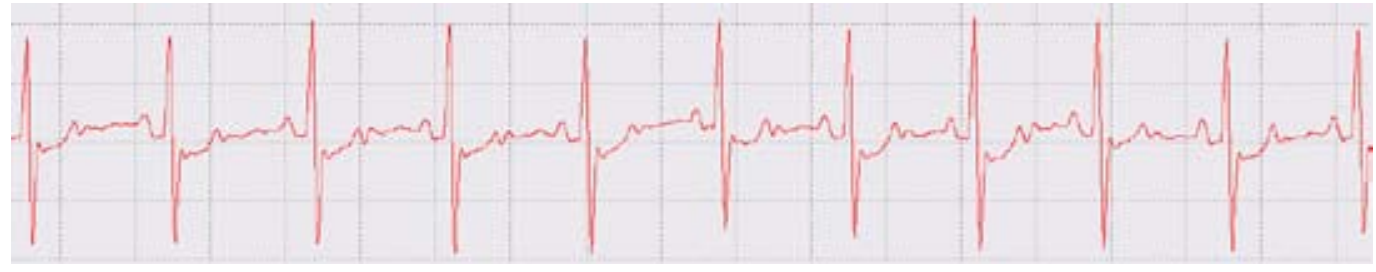

6

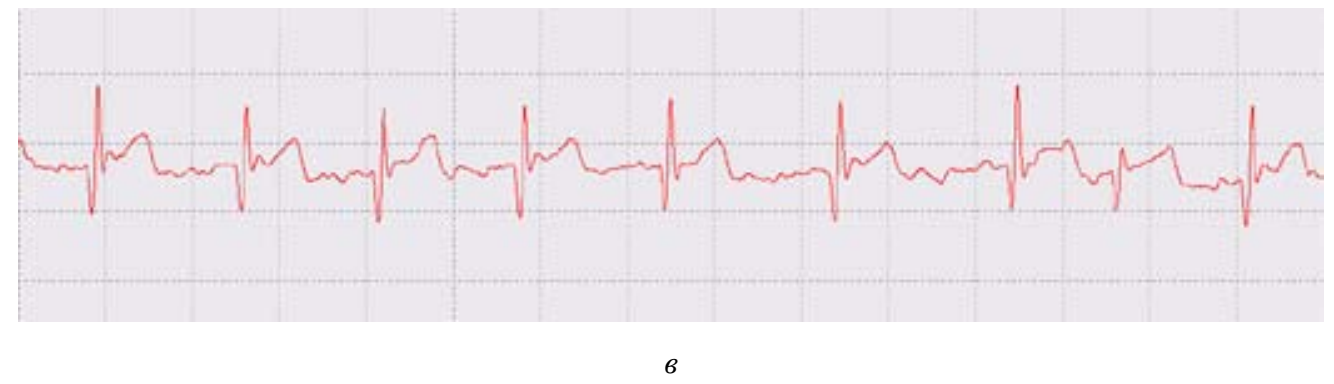

Рис. 5. ЭКС в норме - $a$, при инфаркте миокарда $-\sigma$ и при фибрилляции предсердий - 8

Fig. 5. ECS in norm (a), at a myocardial infarction ( $(\sigma)$, and at a fibrillation of auricles $(\theta)$ 

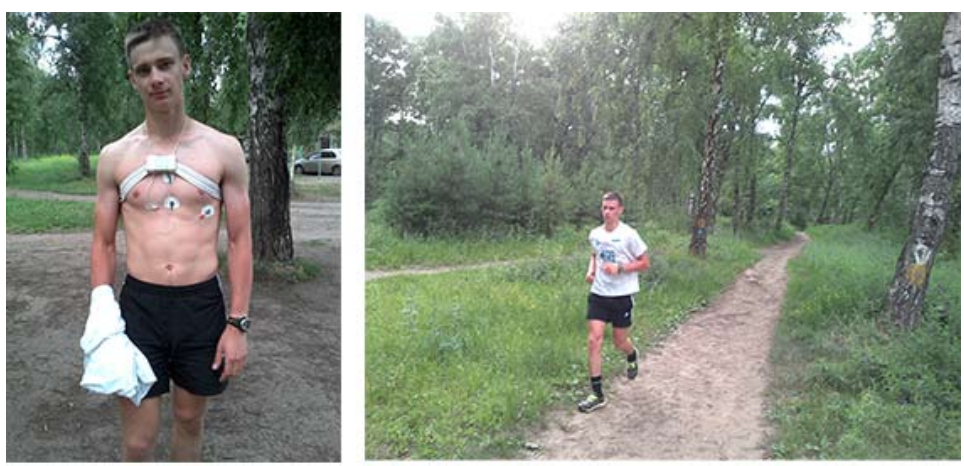

Рис. 6. АПК ДМ на базе МКМ-11 для исследования ССД на спортивной тренировке Fig. 6. HSC DM on the basis of MKM-11 for the study of CVS in sports training

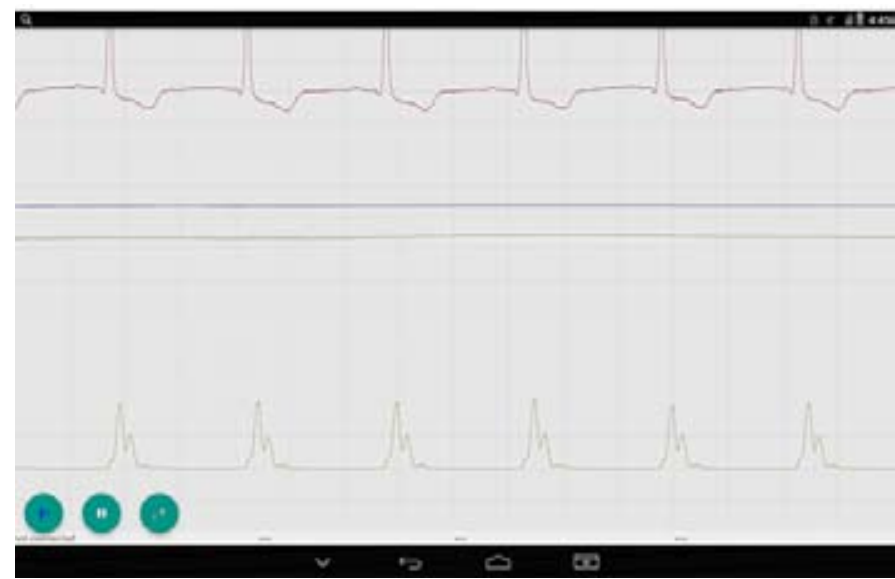

Рис. 7. Результаты эксперимента в спортивной медицине на экране смартфона

Fig. 7. Results of the experiment in sports medicine on the smartphone display

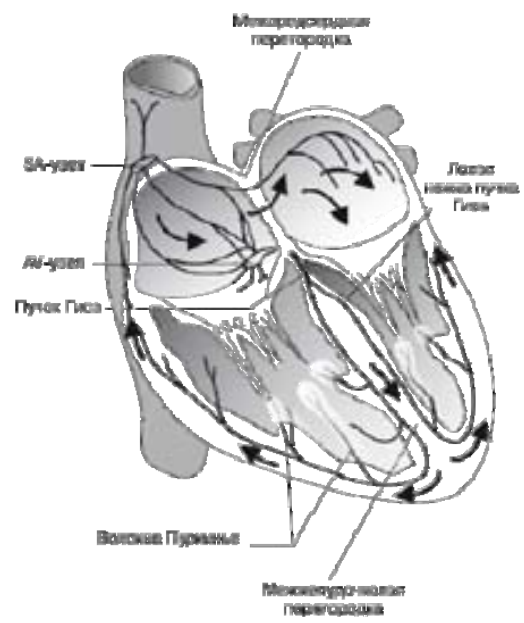

Рис. 8. Фрактальная структура ПНСС, ветвящаяся с самоподобием $1 / f$

Fig. 8. The fractal structure of the $\mathrm{CNNH}$ is ramified with the self-similarity $1 / f$ 

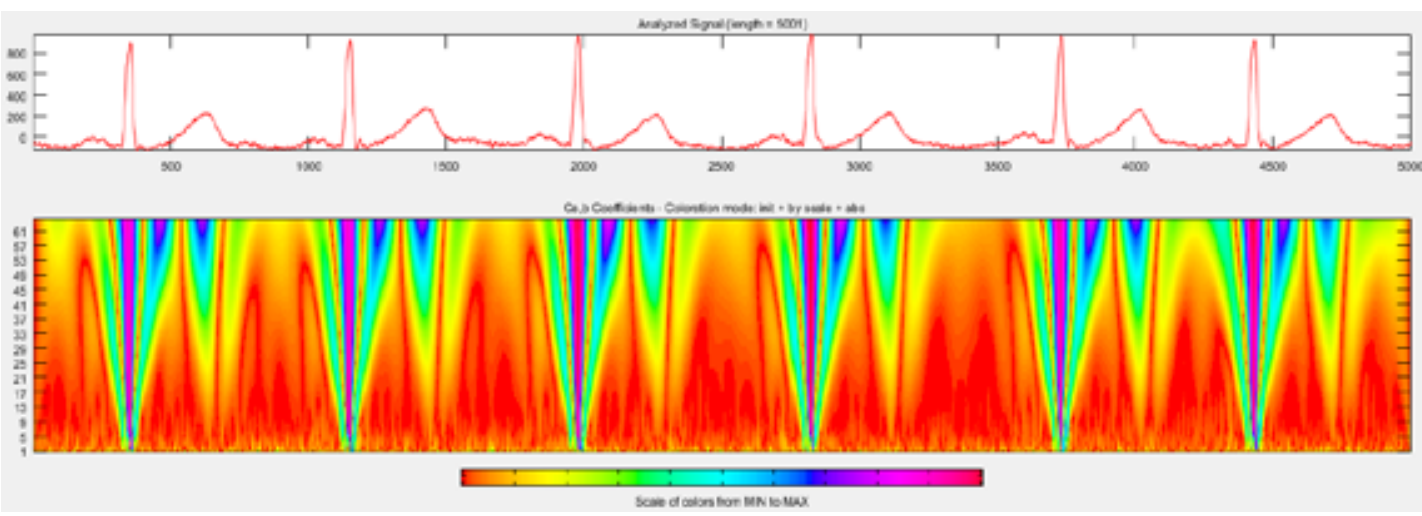

Рис. 9. Вейвлет-преобразование ЭКГ сигнала

Fig. 9. Wavelet transform of ECG signal
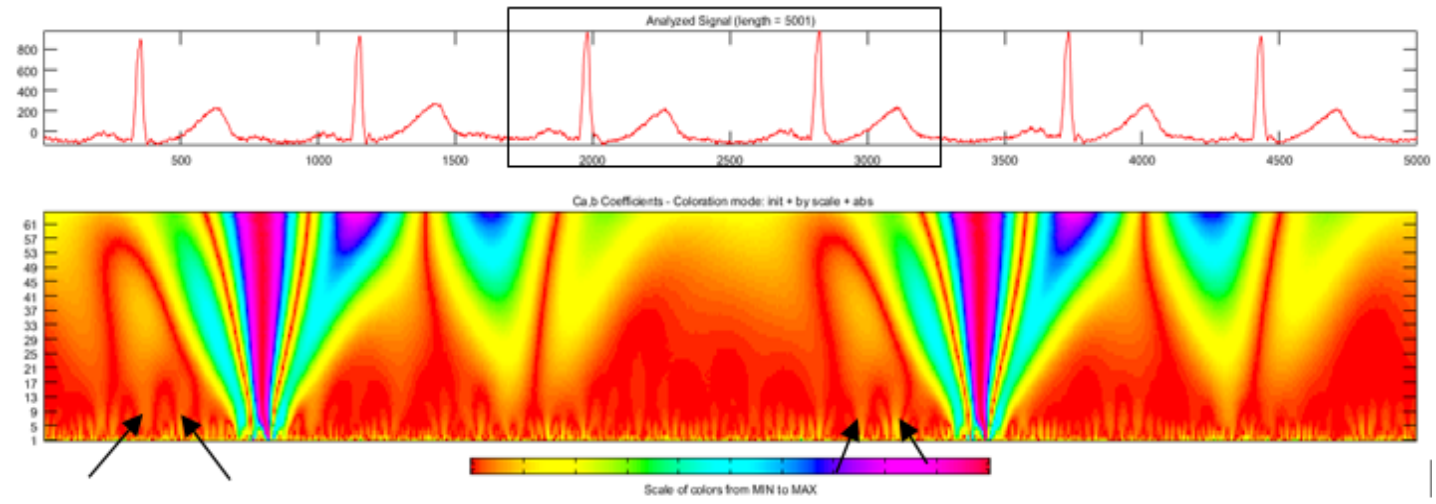

Рис. 10. Выявление латентной структуры ЭКГ-сигнала на вейвлет-диаграмме

Fig. 10. Detection of the latent structure of the ECG signal on the wavelet diagram

ную структуру ЭКС, которая отражает фазово-пространственное похождение возбуждения по сегментам проводящей сети сердца с помощью вейвлет-преобразования.

Выявление латентной структуры ЭКГ-сигнала на вейвлет-диаграмме наблюдается раздельно при прохождении возбуждения в левом, а потом в правом предсердии (рис. 10).

\section{Заключение}

Посредством вейвлет-представления ЭКС возможно отражать фазы распространения возбуждений по проводящей сети, что позволяет увязать наличие или отсутствие возбуждения во всех фрагментах сети. Это дает основание строить диагностику нарушения проводящей системы сердца и сосудистых сетей на основе автоволнового моделирования, анализа вейвлетпреобразований и выявить нарушения в сердечно-сосудистой системе $[5,6]$. 


\section{Список литературы}

[1] Солдатов А.В., Попов А.С., Алдонин Г.М., Андусева В.Г. Структурный топологический анализ проводящей системы сердца. Журнал Сиб. фед. ун-та. Техника и технологии, 2014 (7), 853-856 [Soldatov A.V., Popov A.S., Aldonin G.M. and Andyuseva V.G. Structural Topological Analysis of Cardiac Conduction System, Journal of Siberian Federal University. Engineering \& Technologies, 2014 (7), 853-856 (in Russian)]

[2] Алдонин Г.М. Нелинейные динамические модели и структурный анализ проводящей системы сердца. Успехи современной радиоэлектроники. 2012, (9), 46-50. [Aldonin G.M. Nonlinear dynamic models and structural analysis of the conduction system of the heart, Successes of modern radio electronics, 2012 (9), 46-50 (in Russian)]

[3] Алдонин Г.М. Автономный мониторинг комплекса параметров сердечно-сосудистой системы, Медииинская техника. 2012, (6), 14-18. [Aldonin G.M. Autonomous Monitoring of the Main Set of Parameters of the Cardiovascular System. Biomedical Engineering, 2012 (6), 14-18 (in Russian)]

[4] Autonomous Monitoring of the Main Set of Parameners of the Cardiovascular Sistem, Biomedical Engeeniring, 2013, 46 (6), 232-236.

[5] Мещеряков Р.В., Щипунов Е.Ф., Васильцева О.Я. Оценка взаимосвязей между параметрами сердечно-сосудистой системы человека, Биомедииинская радиоэлектроника. 2013 (5), 014-020 [Meshcheryakov R.V., Shchipunov E.F., Vasiltseva O.Ya. Evaluation of the relationship between the parameters of the cardiovascular system of man, Biomedical radioelectronics. 2013 (5), 14-20 (in Russian)]

[6] Козыренко Н.С., Мещеряков Р.В., Ходашинский И.А., Ануфриева Н.Ю. Математическое и алгоритмическое обеспечение оценки состояния здоровья человека. Труды СПИИРАН. 2014, 2 (33), 116-146 [Kozyrenko N.S., Meshcheryakov R.V., Khodashinsky I.A., Anufrieva N.Yu. Mathematical and algorithmic support for assessing the state of human health. Proceedings of SPIIRAN. 2014, 2 (33), 116-146 (in Russian)] 